\title{
Some Results on Wavelet Frame Packets
}

\author{
Sana Khan, Mohammad Kalimuddin Ahmad \\ Department of Mathematics, Aligarh Muslim University, Aligarh, India \\ Email: sana17khan53@gmail.com, ahmad kalimuddin@yahoo.co.in
}

Received 14 September 2014; revised 18 October 2014; accepted 29 October 2014

Copyright (C) 2014 by authors and Scientific Research Publishing Inc.

This work is licensed under the Creative Commons Attribution International License (CC BY).

http://creativecommons.org/licenses/by/4.0/

c) (i) Open Access

\begin{abstract}
The aim of this paper is to study wavelet frame packets in which there are many frames. It is a generalization of wavelet packets. We derive few results on wavelet frame packets and have obtained the corresponding frame bounds.
\end{abstract}

\section{Keywords}

\section{Wavelet, Wavelet Packets, Frame Packets}

\section{Introduction}

Let us consider an orthonormal wavelet of $L^{2}(\mathbb{R})$. The orthonormal wavelet bases $\left\{\psi_{j, k}: j, k \in \mathbb{Z}\right\}$ have a frequency localization which is proportional to $2^{j}$ at the resolution level $j$. If we consider a bandlimited wavelet $\psi$ (i.e. $\hat{\psi}$ is compactly supported), the measure of supp $\left(\hat{\psi}_{j, k}\right)$ is $2^{j}$ times the measure of supp $(\hat{\psi})$, since

$$
\left(\hat{\psi}_{j, k}\right)(\omega)=2^{-j / 2} \hat{\psi}\left(2^{-j} \omega\right) \mathrm{e}^{-i\left(\frac{\omega}{2^{j}}\right) k}, \quad j, k \in \mathbb{Z},
$$

where $\psi_{j, k}=2^{j / 2} \psi\left(2^{j} x-k\right)$. The wavelet bases have poor frequency localization when $j$ is large. For some applications, it is more convenient to have orthonormal bases with better frequency localization. This will be provided by the wavelet packets.

The wavelet packets introduced by Coifman, Meyer and Wickerhauser [1] [2] played an important role in the applications of wavelet analysis. But the theory itself is worthy for further study. Some developments in the wavelet packet theory should be mentioned, for instance Shen [3] generalized the notion of univariate orthogonal wavelet packets to the case of multivariate wavelet packets. Chui and $\mathrm{Li}$ [4] generalized the concept of orthogonal wavelet packets to the case of nonorthogonal wavelet packets. Yang [5] constructed a-scale orthogonal multiwavelet packets which were more flexible in applications. In [6], Chen and Cheng studied compactly supported orthogonal vector-valued wavelets and wavelet packets. Other notable generalizations are biorthogonal 
wavelet packets [7] and non-orthogonal wavelet packets with r-scaling functions [8]. For a nice exposition of wavelet packets of $L^{2}(\mathbb{R})$, see [9].

The main tool used in the construction of wavelet packets is the splitting trick [10]. Let $\left\{V_{j}: j \in \mathbb{Z}\right\}$ be an MRA of $L^{2}(\mathbb{R})$ with the corresponding scaling function $\phi$ and the wavelet $\psi$. Let $W_{j}$ be the corresponding wavelet subspaces $W_{j}=\overline{\operatorname{span}}\left\{\psi_{j, k}: k \in \mathbb{Z}\right\}$. In the construction of a wavelet from an MRA, the space $V_{1}$ is split into two orthogonal components $V_{0}$ and $W_{0}$, where $V_{1}$ is the closure of the linear span of the functions $\left\{2^{1 / 2} \phi(2 x-k): k \in \mathbb{Z}\right\}$ and $V_{0}$ and $W_{0}$ are the closure of the span of $\{\phi(x-k): k \in \mathbb{Z}\}$ and $\{\psi(x-k): k \in \mathbb{Z}\}$ respectively. Since $\phi(2 x-k)=\phi\left(2\left(x-\frac{k}{2}\right)\right)$, we see that the above procedure splits the half integer translates of a function into the integer translates of two functions.

We can also choose to split $W_{j}$ which is the span of $\left\{\psi\left(2^{j} x-k\right): k \in \mathbb{Z}\right\}=\left\{\psi\left(2^{j}\left(x-\frac{k}{2^{j}}\right)\right): k \in \mathbb{Z}\right\}$. We then have two functions whose $2^{-(j-1)} k$ translates will span the same space $W_{j}$. Repeating the splitting procedure $j$ times, we get $2^{j}$ functions whose integer translates alone span the space $W_{j}$. If we apply this to each $W_{j}$, then the resulting basis of $L^{2}(\mathbb{R})$ will give us a better frequency localization. This basis is called "wavelet packet basis".

There are many orthonormal bases in the wavelet packets. Efficient algorithms for finding the best possible basis do exist; however for certain wavelet applications in signal analysis, frames are more suitable than orthonormal bases, due to the redundancy in frames. Therefore, it is worthwhile to generalize the construction of wavelet packets to wavelet frame packets in which there are many frames. The wavelet frame packets on $\mathbb{R}$ was studied in [11], and the frame packets on $\mathbb{R}^{d}$ were studied by Long and Chen in [12] [13]. Also, multiwavelet packets and frame packets of $L^{2}\left(\mathbb{R}^{d}\right)$ were discussed in [14].

Throughout the paper, the space of all square integrable functions on the real line will be denoted by $L^{2}(\mathbb{R})$ and the inner product and Fourier transform of functions in $L^{2}(\mathbb{R})$ is given by

$$
\langle f, g\rangle=\int_{-\infty}^{\infty} f(x) \overline{g(x)} \mathrm{d} x
$$

and

$$
\hat{f}(\omega):=\int_{-\infty}^{\infty} f(x) \mathrm{e}^{-i \omega x} \mathrm{~d} x
$$

respectively. Also the norm of any $f$ in $L^{2}(\mathbb{R})$ will be denoted by $\|f\|=\langle f, f\rangle^{1 / 2}$ and the relationship between functions and their Fourier transform is defined by $2 \pi\langle f, g\rangle=\langle\hat{f}, \hat{g}\rangle$. For $f \in L^{1}(\mathbb{R}) \cap L^{2}(\mathbb{R})$, the Fourier transform $\hat{f}$ of $f$ is in $L^{2}(\mathbb{R})$ and satisfies the Parseval identity $\|\hat{f}\|_{2}^{2}=2 \pi\|f\|_{2}^{2}$. Also, let $L^{\infty}(\mathbb{R})$ be the collection of almost everywhere (a.e.) bounded functions, i.e., functions bounded everywhere except on sets of (Lebesgue) measure zero and equipped with the norm

$$
\|f\|_{\infty}=\operatorname{ess} \sup _{-\infty<x<\infty}|f(x)| \text {. }
$$

\section{Wavelet Packets and Wavelet Frame Packets}

Definition 1. A multiresolution analysis (MRA) consists of a sequence of closed subspaces $V_{j}, j \in \mathbb{Z}$ of $L^{2}(\mathbb{R})$ and a function $\phi \in V_{0}$, such that the following conditions hold:

1) $\cdots V_{-1} \subset V_{0} \subset V_{1} \cdots$.

2) $\overline{\bigcup_{j \in \mathbb{Z}} V_{j}}=L^{2}(\mathbb{R})$ and $\bigcap_{j \in \mathbb{Z}} V_{j}=\{0\}$.

3) $f(x) \in V_{j} \Leftrightarrow f(2 x) \in V_{j+1}, \quad \forall j \in \mathbb{Z}$.

4) $f \in V_{0} \Rightarrow T_{k} f \in V_{0}, \quad \forall k \in \mathbb{Z}$.

5) $\left\{T_{k} \phi\right\}_{k \in \mathbb{Z}}$ is an orthonormal basis for $V_{0}$. 
The function $\phi$ is called the scaling function of the given MRA.

Suppose that $\phi$ generates a multiresolution analysis and that there exists some function $\psi$ in $L^{2}(\mathbb{R})$ such that $W_{0}=\overline{\operatorname{span}}\{\psi(x-k): k \in \mathbb{Z}\}$ is the orthogonal complement of $V_{0}$ in $V_{1}$. Then $\psi$ is called a basic wavelet relative to $\phi$.

If $\psi$ is a basic wavelet relative to $\phi$, then it is clear that the wavelet spaces $W_{j}$ generated by $\psi$, satisfy the following properties:

6) $V_{j+1}=V_{j} \oplus W_{j}, \quad \forall j \in \mathbb{Z}$.

7) $W_{j} \perp W_{k}, \quad \forall k \neq j$.

8) $L^{2}(\mathbb{R})=\oplus W_{j}$.

Since both the scaling function $\phi \in V_{0}$ and the wavelet $\psi \in W_{0}$ are in $V_{1}$ and $V_{1}$ is generated by $\phi_{1, k}(x)=\sqrt{2} \phi(2 x-k)$, there exists two sequences $\left\{p_{k}\right\}$ and $\left\{q_{k}\right\}$ in $\ell^{2}$ such that

$$
\begin{aligned}
& \phi(x)=\sum_{k \in \mathbb{Z}} p_{k} \phi_{1, k}(x)=\sqrt{2} \sum_{k \in \mathbb{Z}} p_{k} \phi(2 x-k), \\
& \psi(x)=\sum_{k \in \mathbb{Z}} q_{k} \phi_{1, k}(x)=\sqrt{2} \sum_{k \in \mathbb{Z}} q_{k} \phi(2 x-k),
\end{aligned}
$$

for all $x \in \mathbb{R}$. For the Haar basis, we have

$$
\begin{aligned}
& p_{0}=\frac{1}{\sqrt{2}}, \quad p_{1}=\frac{1}{\sqrt{2}} \\
& q_{0}=\frac{1}{\sqrt{2}}, \quad q_{1}=-\frac{1}{\sqrt{2}} .
\end{aligned}
$$

Therefore, for the Haar basis, the scaling function and the wavelet function satisfy the following recurrence equation

$$
\begin{array}{r}
\phi(x)=\phi(2 x)+\phi(2 x-1) \\
\psi(x)=\phi(2 x)-\phi(2 x-1) .
\end{array}
$$

Due to Coifman, Meyer and Wickerhauser [1] [2], we have the following sequences of functions

$$
\begin{aligned}
& \mathcal{W}_{2 n}(x)=\sqrt{2} \sum_{k \in \mathbb{Z}} a_{k} \mathcal{W}_{n}(2 x-k) \\
& \mathcal{W}_{2 n+1}(x)=\sqrt{2} \sum_{k \in \mathbb{Z}} b_{k} \mathcal{W}_{n}(2 x-k),
\end{aligned}
$$

where $n=0,1,2, \cdots$ and $a=\left\{a_{k}\right\}$ is the filter which satisfies the following properties

$$
\sum_{n \in \mathbb{Z}} a_{n-2 k} \cdot a_{n-2 l}=\delta_{k, l}, \quad \sum_{n \in \mathbb{Z}} a_{n}=\sqrt{2},
$$

where $\delta_{k, l}$ is the Kronecker delta defined by

$$
\delta_{k, l}= \begin{cases}0 & \text { if } k \neq l \\ 1 & \text { if } k=l\end{cases}
$$

and

$$
b_{k}=(-1)^{k} a_{1-k}
$$

For $n=0$ in (7) and (8), we get

$$
\begin{aligned}
& \mathcal{W}_{0}(x)=\mathcal{W}_{0}(2 x)+\mathcal{W}_{0}(2 x-1) \\
& \mathcal{W}_{1}(x)=\mathcal{W}_{0}(2 x)-\mathcal{W}_{0}(2 x-1)
\end{aligned}
$$

$\mathcal{W}_{0}(x)$ corresponds to our scaling function $\phi$ and $\mathcal{W}_{1}(x)$ corresponds to the wavelet $\psi$. If we increase $n$, we get the following structures 


$$
\begin{aligned}
& \mathcal{W}_{2}(x)=\mathcal{W}_{1}(2 x)+\mathcal{W}_{1}(2 x-1), \\
& \mathcal{W}_{3}(x)=\mathcal{W}_{1}(2 x)-\mathcal{W}_{1}(2 x-1), \\
& \mathcal{W}_{4}(x)=\mathcal{W}_{1}(4 x)+\mathcal{W}_{1}(4 x-1)+\mathcal{W}_{1}(4 x-2)+\mathcal{W}_{1}(4 x-3),
\end{aligned}
$$

and so on. The functions $\mathcal{W}_{m}, m=2 n$ or $2 n+1, n=0,1,2, \cdots$ are called "wavelet packets" relative to the scaling function $\mathcal{W}_{0}=\phi$. Thus, the family $\left\{\mathcal{W}_{m}\right\}$ is a generalization of the wavelet $\mathcal{W}_{1}=\psi$.

Definition 2. The family $\left\{2^{j / 2} \mathcal{W}_{n}\left(2^{j} x-k\right)\right\}, n, j \in \mathbb{Z}_{+}, k \in \mathbb{Z}$ is called a wavelet basis packet, where $n$ is the oscillation parameter, $j$ the scaling parameter and $k$ the translation parameter.

We can also write $\mathcal{W}_{n ; j, k}=2^{j / 2} \mathcal{W}_{n}\left(2^{j} x-k\right)$. The family $\left\{\mathcal{W}_{n ; j, k}\right\}$ constitutes wavelet frame packets if there are constants $\mathcal{K}$ and $\mathcal{L}, 0<\mathcal{K} \leq \mathcal{L}<\infty$ such that

$$
\mathcal{K}\|f\|^{2} \leq \sum_{n} \sum_{j, k \in \mathbb{Z}}\left|\left\langle f, \mathcal{W}_{n ; j, k}\right\rangle\right|^{2} \leq \mathcal{L}\|f\|^{2}, \quad \forall f \in L^{2}(\mathbb{R}) .
$$

\section{Main Results}

Define $\alpha_{r}(\omega)=\sum_{n} \sum_{j=0}^{\infty} \hat{\mathcal{W}}_{n}\left(2^{j} \omega\right) \overline{\hat{\mathcal{W}}_{n}\left(2^{j}(\omega+2 r \pi)\right)}, \omega \in \mathbb{R}, \quad r \in \mathbb{Z}$ and

$$
\mathcal{H}(\omega)=\sum_{n} \sum_{j \in \mathbb{Z}}\left|\hat{\mathcal{W}}_{n}\left(2^{-j} \omega\right)\right|^{2}, \quad \omega \in \mathbb{R} .
$$

Consider

$$
\mathcal{H}_{\mathcal{W}}^{-}=\operatorname{ess} \inf _{\omega \in \mathbb{R}} \mathcal{H}(\omega), \quad H_{\mathcal{W}}^{+}=\operatorname{ess} \sup _{\omega \in \mathbb{R}} \mathcal{H}(\omega)
$$

and

$$
\delta_{\mathcal{W}}(r)=\operatorname{ess} \sup _{\omega \in \mathbb{R}} \sum_{k \in \mathbb{Z}}\left|\alpha_{r}\left(2^{k} \omega\right)\right| .
$$

Theorem 1. Let $\left\{\mathcal{W}_{n}\right\}$ be the basic wavelet packets such that

$$
\mathcal{K}=\mathcal{H}_{\mathcal{W}}^{-}-\sum_{\mu \in 2 \mathbb{Z}+1} \sqrt{\delta_{\mathcal{W}}(\mu) \delta_{\mathcal{W}}(-\mu)}>0,
$$

and

$$
\mathcal{L}=\mathcal{H}_{\mathcal{W}}^{+}+\sum_{\mu \in 2 \mathbb{Z}+1} \sqrt{\delta_{\mathcal{W}}(\mu) \delta_{\mathcal{W}}(-\mu)}<\infty .
$$

Then $\left\{\mathcal{W}_{n ; j, k}\right\}$ constitutes wavelet frame packets with frame bounds $\mathcal{K}$ and $\mathcal{L}$.

Proof. Let $\mathcal{D}$ be the class of all those functions $f \in L^{2}(\mathbb{R})$ such that $\hat{f} \in L^{\infty}(\mathbb{R})$ and $\hat{f}$ is compactly supported in $\mathbb{R} \backslash 0$. By using the Parseval identity, we have

$$
\left\langle f, \mathcal{W}_{n ; j, k}\right\rangle=\frac{1}{2 \pi}\left\langle\hat{f}, \hat{\mathcal{W}}_{n ; j, k}\right\rangle=\frac{1}{2 \pi} \int_{-\infty}^{\infty} \hat{f}(\omega) \overline{\mathcal{W}}_{n ; j, k}(\omega) \mathrm{d} \omega .
$$

Since, $\hat{\mathcal{W}}_{n ; j, k}(\omega)=2^{-j / 2} \hat{\mathcal{W}}_{n}\left(2^{-j} \omega\right) \mathrm{e}^{-i\left(\frac{\omega}{2^{j}}\right) k}$, we have

$$
\begin{aligned}
\left\langle f, \mathcal{W}_{n ; j, k}\right\rangle & =\frac{1}{2 \pi} 2^{-j / 2} \int_{-\infty}^{\infty} \hat{f}(\omega) \overline{\hat{\mathcal{W}}_{n}\left(2^{-j} \omega\right)} \mathrm{e}^{i\left(\frac{\omega}{2^{j}}\right) k} \mathrm{~d} \omega \\
& =\frac{1}{2 \pi} 2^{-j / 2} \int_{-\infty}^{\infty} 2^{j} \hat{f}\left(2^{j} \omega\right) \overline{\hat{\mathcal{W}}_{n}(\omega)} \mathrm{e}^{i k \omega} \mathrm{d} \omega \\
& =\frac{1}{2 \pi} 2^{j / 2} \int_{-\infty}^{\infty} \hat{f}\left(2^{j} \omega\right) \overline{\hat{\mathcal{W}}_{n}(\omega)} \mathrm{e}^{i k \omega} \mathrm{d} \omega .
\end{aligned}
$$

Hence, 


$$
\sum_{n} \sum_{j, k \in \mathbb{Z}}\left|\left\langle f, \mathcal{W}_{n ; j, k}\right\rangle\right|^{2}=\frac{1}{(2 \pi)^{2}} \sum_{n} \sum_{j \in \mathbb{Z}} \sum_{k \in \mathbb{Z}} 2^{j}\left|\int_{\mathbb{R}} \hat{f}\left(2^{j} \omega\right) \overline{\hat{\mathcal{W}}_{n}(\omega)} \mathrm{e}^{i k \omega} \mathrm{d} \omega\right|^{2} .
$$

Let $F_{j}(\omega)=\hat{f}\left(2^{j} \omega\right) \overline{\hat{\mathcal{W}}_{n}(\omega)}$ for $j, n \in \mathbb{Z}$. Each $F_{j}$ is compactly supported in $\mathbb{R} \backslash 0$ and belongs to $L^{2}(\mathbb{R})$. If $F$ is such a function,

$$
\sum_{k \in \mathbb{Z}} F(\omega+2 k \pi)
$$

which is $2 \pi$-periodic and whose Fourier coefficients are $\frac{1}{2 \pi} \hat{F}(k), k \in \mathbb{Z}$, then by Poisson sum formula we have,

$$
\sum_{k \in \mathbb{Z}} F(\omega+2 k \pi)=\frac{1}{2 \pi} \sum_{k \in \mathbb{Z}} \hat{F}(k) \mathrm{e}^{i k \omega} .
$$

Hence,

$$
\frac{1}{2 \pi} \int_{\mathbb{R}} \overline{F(\omega)} \sum_{k \in \mathbb{Z}} \hat{F}(k) \mathrm{e}^{i k \omega} \mathrm{d} \omega=\int_{\mathbb{R}} \overline{F(\omega)} \sum_{k \in \mathbb{Z}} F(\omega+2 k \pi) \mathrm{d} \omega .
$$

But the left side of (13) equals

$$
\frac{1}{2 \pi} \sum_{k \in \mathbb{Z}} \hat{F}(k) \int_{\mathbb{R}} \overline{F(\omega) \mathrm{e}^{-i k \omega} \mathrm{d} \omega}=\frac{1}{2 \pi} \sum_{k \in \mathbb{Z}}|\hat{F}(k)|^{2} .
$$

It follows that

$$
\frac{1}{2 \pi} \sum_{k \in \mathbb{Z}}|\hat{F}(k)|^{2}=\int_{\mathbb{R}} \overline{F(\omega)} \sum_{k \in \mathbb{Z}} F(\omega+2 k \pi) \mathrm{d} \omega .
$$

Applying (15) when $F=F_{j}$ in (12) we obtain

$$
\begin{aligned}
\sum_{n} \sum_{j, k \in \mathbb{Z}}\left|\left\langle f, \mathcal{W}_{n ; j, k}\right\rangle\right|^{2} & =\frac{1}{2 \pi} \sum_{n} \sum_{j \in \mathbb{Z}} 2^{j} \int_{\mathbb{R}} \overline{\hat{f}\left(2^{j} \omega\right)} \hat{\mathcal{W}}_{n}(\omega) \sum_{k \in \mathbb{Z}} \hat{f}\left(2^{j}(\omega+2 k \pi)\right) \overline{\hat{\mathcal{W}}_{n}(\omega+2 k \pi)} \mathrm{d} \omega \\
& =\frac{1}{2 \pi} \sum_{n} \sum_{j \in \mathbb{Z}} 2^{j} \int_{\mathbb{R}} \overline{\hat{f}\left(2^{j} \omega\right)} \hat{\mathcal{W}}_{n}(\omega)\left\{\hat{f}\left(2^{j} \omega\right) \overline{\hat{\mathcal{W}}_{n}(\omega)}+\sum_{k \neq 0} \hat{f}\left(2^{j}(\omega+2 k \pi)\right) \overline{\hat{\mathcal{W}}_{n}(\omega+2 k \pi)}\right\} \mathrm{d} \omega \\
& =\frac{1}{2 \pi} \int_{\mathbb{R}}|\hat{f}(\omega)|^{2} \sum_{n} \sum_{j \in \mathbb{Z}}\left|\hat{\mathcal{W}}_{n}\left(2^{-j} \omega\right)\right|^{2} \mathrm{~d} \omega+I,
\end{aligned}
$$

where,

$$
I=\frac{1}{2 \pi} \sum_{n} \sum_{j \in \mathbb{Z}} 2^{j} \int_{\mathbb{R}} \overline{\hat{f}\left(2^{j} \omega\right)} \hat{\mathcal{W}}_{n}(\omega) \sum_{k \neq 0} \hat{f}\left(2^{j}(\omega+2 k \pi)\right) \overline{\hat{\mathcal{W}}_{n}(\omega+2 k \pi)} \mathrm{d} \omega .
$$

In the expression for $I$, the parameter $k$ is a non-zero integer. For each such $k$ there is a unique nonnegative integer $l$ and a unique odd integer $q$ such that $k=2^{l} q$. Therefore, we have

$$
\begin{aligned}
2 \pi I & =\sum_{n} \sum_{j \in \mathbb{Z}} \int_{\mathbb{R}} \overline{\hat{f}(\omega)} \hat{\mathcal{W}}_{n}\left(2^{-j} \omega\right) \sum_{k \neq 0} \hat{f}\left(\omega+2^{j} 2 k \pi\right) \overline{\hat{\mathcal{W}}_{n}\left(2^{-j} \omega+2 k \pi\right)} \mathrm{d} \omega \\
& =\sum_{n} \sum_{j \in \mathbb{Z}} \int_{\mathbb{R}} \overline{\hat{f}(\omega)} \hat{\mathcal{W}}_{n}\left(2^{-j} \omega\right) \sum_{l=0}^{\infty} \sum_{q \in 2 \mathbb{Z}+1} \hat{f}\left(\omega+2^{j+l} 2 q \pi\right) \overline{\hat{\mathcal{W}}_{n}\left(2^{l}\left(2^{-j-l} \omega+2 q \pi\right)\right)} \mathrm{d} \omega \\
& =\int_{\mathbb{R}} \overline{\hat{f}(\omega)} \sum_{q \in 2 \mathbb{Z}+1 l=0} \sum_{p \in \mathbb{Z}}^{\infty} \sum_{n} \hat{f}\left(\omega+2^{p} 2 q \pi\right) \hat{\mathcal{W}}_{n}\left(2^{l} 2^{-p} \omega\right) \overline{\hat{\mathcal{W}}_{n}\left(2^{l}\left(2^{-p} \omega+2 q \pi\right)\right)} \mathrm{d} \omega \\
& =\int_{\mathbb{R}} \overline{\hat{f}(\omega)} \sum_{q \in 2 \mathbb{Z}+1} \sum_{p \in \mathbb{Z}} \hat{f}\left(\omega+2^{p} 2 q \pi\right) \alpha_{q}\left(2^{-p} \omega\right) \mathrm{d} \omega .
\end{aligned}
$$

Thus, 


$$
\begin{aligned}
\sum_{n} \sum_{j, k \in \mathbb{Z}}\left|\left\langle f, \mathcal{W}_{n ; j, k}\right\rangle\right|^{2} & =\frac{1}{2 \pi} \int_{\mathbb{R}}|\hat{f}(\omega)|^{2} \sum_{n} \sum_{j \in \mathbb{Z}}\left|\hat{\mathcal{W}}_{n}\left(2^{-j} \omega\right)\right|^{2} \mathrm{~d} \omega+\frac{1}{2 \pi} \int_{\mathbb{R}} \overline{\hat{f}(\omega)} \sum_{q \in 2 \mathbb{Z}+1 p \in \mathbb{Z}} \sum_{\hat{f}}\left(\omega+2^{p} 2 q \pi\right) \alpha_{q}\left(2^{-p} \omega\right) \mathrm{d} \omega \\
& =\frac{1}{2 \pi} \int_{\mathbb{R}}|\hat{f}(\omega)|^{2} \sum_{n} \sum_{j \in \mathbb{Z}}\left|\hat{\mathcal{W}}_{n}\left(2^{-j} \omega\right)\right|^{2} \mathrm{~d} \omega+\frac{1}{2 \pi} \mathcal{P}(f),
\end{aligned}
$$

for all $f \in \mathcal{D}$. By using Schwarz's inequality we have

$$
|\mathcal{P}(f)| \leq \sum_{q \in 2 \mathbb{Z}+1} \sum_{p \in \mathbb{Z}}\left(\int_{\mathbb{R}}|\hat{f}(\eta)|^{2}\left|\alpha_{q}\left(2^{-p} \eta\right)\right| \mathrm{d} \eta\right)^{1 / 2} \cdot\left(\int_{\mathbb{R}}\left|\hat{f}\left(\eta+2^{p} 2 q \pi\right)\right|^{2}\left|\alpha_{q}\left(2^{-p} \eta\right)\right| \mathrm{d} \eta\right)^{1 / 2} .
$$

By changing variables in the second integral and using the fact that $\alpha_{q}(\omega-2 q \pi)=\overline{\alpha_{-q}(\omega)}$, and applying Schwarz's inequality for series we have

$$
\begin{aligned}
|\mathcal{P}(f)| & \leq \sum_{q \in 2 \mathbb{Z}+1}\left(\sum_{p \in \mathbb{Z}} \int_{\mathbb{R}}|\hat{f}(\eta)|^{2}\left|\alpha_{q}\left(2^{-p} \eta\right)\right| \mathrm{d} \eta\right)^{1 / 2} \cdot\left(\sum_{p \in \mathbb{Z}} \int_{\mathbb{R}}|\hat{f}(\eta)|^{2}\left|\alpha_{-q}\left(2^{-p} \eta\right)\right| \mathrm{d} \eta\right)^{1 / 2} \\
& \leq \sum_{q \in 2 \mathbb{Z}+1}\left[\delta_{\mathcal{W}}(q) \delta_{\mathcal{W}}(-q)\right]^{1 / 2}\|\hat{f}\|_{2}^{2} .
\end{aligned}
$$

Hence,

$$
-\sum_{q \in 2 \mathbb{Z}+1}\left[\delta_{\mathcal{W}}(q) \delta_{\mathcal{W}}(-q)\right]^{1 / 2}\|\hat{f}\|_{2}^{2} \leq \mathcal{P}(f) \leq \sum_{q \in 2 \mathbb{Z}+1}\left[\delta_{\mathcal{W}}(q) \delta_{\mathcal{W}}(-q)\right]^{1 / 2}\|\hat{f}\|_{2}^{2} .
$$

These inequalities together with (16) give us

$$
\mathcal{K}\|f\|_{2}^{2} \leq \sum_{n} \sum_{j, k \in \mathbb{Z}}\left|\left\langle f, \mathcal{W}_{n ; j, k}\right\rangle\right|^{2} \leq \mathcal{L}\|f\|_{2}^{2}, \quad \forall f \in \mathcal{D} .
$$

Since $\mathcal{D}$ is dense in $L^{2}(\mathbb{R})$, the above inequality holds for all $f \in L^{2}(\mathbb{R})$.

Theorem 2. The system $\left\{\mathcal{W}_{n, j, k}\right\}, n, j \in \mathbb{Z}_{+}, k \in \mathbb{Z}$ is orthonormal if and only if

$$
\sum_{n} \sum_{k \in \mathbb{Z}}\left|\hat{\mathcal{W}}_{n}(\omega+2 k \pi)\right|^{2}=1 \text { for a.e. } \omega \in \mathbb{R}
$$

and

$$
\sum_{n} \sum_{k \in \mathbb{Z}} \hat{\mathcal{W}}_{n}\left(2^{j}(\omega+2 k \pi)\right) \overline{\hat{\mathcal{W}}_{n}(\omega+2 k \pi)}=0 \text { for a.e. } \omega \in \mathbb{R}, \quad j \geq 1
$$

Proof. By using the Plancherel theorem we have

$$
\begin{aligned}
\delta_{k, 0} & =\sum_{n} \int_{\mathbb{R}} \mathcal{W}_{n}(x) \overline{\mathcal{W}_{n}(x-k)} \mathrm{d} x \\
& =\frac{1}{2 \pi} \int_{\mathbb{R}} \sum_{n}\left|\hat{\mathcal{W}}_{n}(\omega)\right|^{2} \mathrm{e}^{i k \omega} \mathrm{d} \omega \\
& =\frac{1}{2 \pi} \sum_{l=-\infty}^{\infty} \int_{2 l \pi}^{2(l+1) \pi} \sum_{n}\left|\hat{\mathcal{W}}_{n}(\omega)\right|^{2} \mathrm{e}^{i k \omega} \mathrm{d} \omega \\
& =\frac{1}{2 \pi} \sum_{l=-\infty}^{\infty} \int_{0}^{2 \pi} \sum_{n}\left|\hat{\mathcal{W}}_{n}(\mu+2 l \pi)\right|^{2} \mathrm{e}^{i k \mu} \mathrm{d} \mu \\
& =\frac{1}{2 \pi} \int_{0}^{2 \pi}\left(\sum_{n} \sum_{l \in \mathbb{Z}}\left|\hat{\mathcal{W}}_{n}(\mu+2 l \pi)\right|^{2}\right) \mathrm{e}^{i k \mu} \mathrm{d} \mu .
\end{aligned}
$$

Thus, $\left\{\mathcal{W}_{n ; 0, k}\right\}$ is orthonormal if and only if $\sum_{n} \sum_{l \in \mathbb{Z}}\left|\hat{\mathcal{W}}_{n}(\mu+2 l \pi)\right|^{2}=1$ a.e. The converse is immediate. Performing a change of variables, we see that $\left\langle\mathcal{W}_{n ; j, k}, \mathcal{W}_{n ; j, l}\right\rangle=\left\langle\mathcal{W}_{n ; 0, k}, \mathcal{W}_{n ; 0, l}\right\rangle$; this tells us that the system 
$\left\{\mathcal{W}_{n, j, k}\right\}$ is orthonormal for each fixed $j$ when (17) is satisfied. The proof of condition (18) is similar. 口

Lemma 1. If $\left\{\mathcal{W}_{n ; j, k}\right\}$ is an orthonormal system, then

$$
\sum_{n} \hat{\mathcal{W}}_{n}\left(2^{m} \omega\right)=\sum_{n} \sum_{j=1}^{\infty} \sum_{k \in \mathbb{Z}} \hat{\mathcal{W}}_{n}\left(2^{m}(\omega+2 k \pi)\right) \overline{\mathcal{W}}_{n}\left(2^{j}(\omega+2 k \pi)\right) \hat{\mathcal{W}}_{n}\left(2^{j} \omega\right) \text { a.e. }
$$

for all $m \geq 1$.

Proof. Let $A_{m}(\omega)$ be the R.H.S of (19). We have to show that $A_{m}(\omega)=\sum_{n} \hat{\mathcal{W}}_{n}\left(2^{m} \omega\right)$ for a.e. $\omega \in \mathbb{R}$. We first show that $A_{m}(\omega)=A_{m-1}(2 \omega)$ and then that $A_{1}(\omega)=\sum_{n} \hat{\mathcal{W}}_{n}(2 \omega)$; this will clearly give us (19). Using (18), with $j$ replaced by $m$, we have

$$
\begin{aligned}
A_{m}(\omega) & =\sum_{n} \sum_{k \in \mathbb{Z}} \hat{\mathcal{W}}_{n}\left(2^{m}(\omega+2 k \pi)\right) \sum_{j=1}^{\infty} \overline{\hat{\mathcal{W}}_{n}\left(2^{j}(\omega+2 k \pi)\right)} \hat{\mathcal{W}}_{n}\left(2^{j} \omega\right) \\
& =\sum_{n} \sum_{k \in \mathbb{Z}} \hat{\mathcal{W}}_{n}\left(2^{m}(\omega+2 k \pi)\right) \overline{\hat{\mathcal{W}}_{n}(\omega+2 k \pi)} \hat{\mathcal{W}}_{n}(\omega)+\sum_{n} \sum_{k \in \mathbb{Z}} \hat{\mathcal{W}}_{n}\left(2^{m}(\omega+2 k \pi)\right) \sum_{j=1}^{\infty} \overline{\hat{\mathcal{W}}_{n}\left(2^{j}(\omega+2 k \pi)\right)} \hat{\mathcal{W}}_{n}\left(2^{j} \omega\right) \\
& =\sum_{n} \sum_{k \in \mathbb{Z}} \hat{\mathcal{W}}_{n}\left(2^{m}(\omega+2 k \pi)\right) \sum_{j=0}^{\infty} \overline{\hat{\mathcal{W}}_{n}\left(2^{j}(\omega+2 k \pi)\right)} \hat{\mathcal{W}}_{n}\left(2^{j} \omega\right) .
\end{aligned}
$$

Replacing $k$ by $2 l$, we have

$$
\begin{aligned}
A_{m}(\omega) & =\sum_{n} \sum_{l \in \mathbb{Z}} \hat{\mathcal{W}}_{n}\left(2^{m}(\omega+4 l \pi)\right) \sum_{j=0}^{\infty} \overline{\hat{\mathcal{W}}_{n}\left(2^{j}(\omega+4 l \pi)\right)} \hat{\mathcal{W}}_{n}\left(2^{j} \omega\right) \\
& =\sum_{n} \sum_{l \in \mathbb{Z}} \hat{\mathcal{W}}_{n}\left(2^{m+1}\left(\frac{\omega}{2}+2 l \pi\right)\right) \sum_{j=0}^{\infty} \overline{\hat{\mathcal{W}}_{n}\left(2^{j+1}\left(\frac{\omega}{2}+2 l \pi\right)\right)} \hat{\mathcal{W}}_{n}\left(2^{j+1} \frac{\omega}{2}\right) \\
& =\sum_{n} \sum_{l \in \mathbb{Z}} \hat{\mathcal{W}}_{n}\left(2^{m+1}\left(\frac{\omega}{2}+2 l \pi\right)\right) \sum_{j=1}^{\infty} \overline{\hat{\mathcal{W}}_{n}\left(2^{j}\left(\frac{\omega}{2}+2 l \pi\right)\right)} \hat{\mathcal{W}}_{n}\left(2^{j} \frac{\omega}{2}\right) \\
& =A_{m+1}\left(\frac{\omega}{2}\right)
\end{aligned}
$$
This shows that $A_{m}(\omega)=A_{m-1}(2 \omega)$ a.e. Now, we calculate $A_{1}(\omega)$ and show that $A_{1}(\omega)=\sum_{n} \hat{\mathcal{W}}_{n}(2 \omega)$.
Changing variables in the sum over $j$, we have

$$
\begin{aligned}
A_{1}(\omega) & =\sum_{n} \sum_{k \in \mathbb{Z}} \hat{\mathcal{W}}_{n}(2(\omega+2 k \pi)) \sum_{j=1}^{\infty} \overline{\hat{\mathcal{W}}_{n}\left(2^{j}(\omega+2 k \pi)\right)} \hat{\mathcal{W}}_{n}\left(2^{j} \omega\right) \\
& =\sum_{n} \sum_{k \in \mathbb{Z}} \hat{\mathcal{W}}_{n}(2 \omega+4 k \pi) \sum_{j=0}^{\infty} \overline{\hat{\mathcal{W}}_{n}\left(2^{j}(2 \omega+4 k \pi)\right)} \hat{\mathcal{W}}_{n}\left(2^{j} 2 \omega\right) \\
& =\sum_{n} \sum_{k \in \mathbb{Z}} \hat{\mathcal{W}}_{n}(2 \omega+2 k \pi) \sum_{j=0}^{\infty} \overline{\hat{\mathcal{W}}_{n}\left(2^{j}(2 \omega+2 k \pi)\right)} \hat{\mathcal{W}}_{n}\left(2^{j} 2 \omega\right) \\
& =\sum_{n} \sum_{k \in \mathbb{Z}} \hat{\mathcal{W}}_{n}(2 \omega+2 k \pi)\left\{\overline{\hat{\mathcal{W}}_{n}(2 \omega+2 k \pi)} \hat{\mathcal{W}}_{n}(2 \omega)+\sum_{j=1}^{\infty} \overline{\hat{\mathcal{W}}_{n}\left(2^{j}(2 \omega+2 k \pi)\right)} \hat{\mathcal{W}}_{n}\left(2^{j} 2 \omega\right)\right\}
\end{aligned}
$$

By using (17) and (18), we have

$$
A_{1}(\omega)=\sum_{n} \hat{\mathcal{W}}_{n}(2 \omega)
$$

Theorem 3. Let $\left\{\mathcal{W}_{n}\right\}$ be a sequence of wavelet frame packets with bounds $\mathcal{K}$ and $\mathcal{L}$. Define $\left\{\gamma_{m}\right\}$ by

$$
\gamma_{m}=\sum_{n=1}^{\infty} \lambda_{m n} \mathcal{W}_{n}, \quad m \in \mathbb{N}
$$


If the numbers $\left\{\lambda_{m n}\right\}_{m, n \in \mathbb{N}}$ satisfy the two conditions

$$
\begin{gathered}
l:=\sup _{n} \sum_{p=1}^{\infty}\left|\sum_{m=1}^{\infty} \lambda_{m n} \overline{\lambda_{m p}}\right|<\infty, \\
k:=\inf _{n}\left(\sum_{m=1}^{\infty}\left|\lambda_{m n}\right|^{2}-\sum_{p \neq n}\left|\sum_{m=1}^{\infty} \lambda_{m n} \overline{\lambda_{m p}}\right|\right)>0,
\end{gathered}
$$

then $\left\{\gamma_{m}\right\}_{m=1}^{\infty}$ defined by $(20)$ is a wavelet frame packet with bounds $k \mathcal{K}$ and $I \mathcal{L}$.

Proof. Let $f \in L^{2}(\mathbb{R})$. Then

$$
\begin{aligned}
\sum_{m=1}^{\infty}\left|\left\langle\gamma_{m}, f\right\rangle\right|^{2} & =\sum_{m=1}^{\infty}\left|\left\langle\sum_{n=1}^{\infty} \lambda_{m n} \mathcal{W}_{n}, f\right\rangle\right|^{2}=\sum_{m=1}^{\infty}\left|\sum_{n=1}^{\infty} \lambda_{m n}\left\langle\mathcal{W}_{n}, f\right\rangle\right|^{2} \\
& =\sum_{m=1}^{\infty} \sum_{n=1}^{\infty}\left|\lambda_{m n}\right|^{2}\left|\left\langle\mathcal{W}_{n}, f\right\rangle\right|^{2}+\sum_{m=1}^{\infty} \sum_{n=1}^{\infty} \sum_{p \neq n} \lambda_{m n} \overline{\lambda_{m p}}\left\langle\mathcal{W}_{n}, f\right\rangle\left\langle f, \mathcal{W}_{p}\right\rangle \\
& =R_{1}(f)+R_{2}(f) .
\end{aligned}
$$

By Cauchy-Schwarz inequality, we get

$$
\begin{aligned}
\left|R_{2}(f)\right| & \leq \sum_{n=1}^{\infty} \sum_{p \neq n}\left|\left\langle\mathcal{W}_{n}, f\right\rangle\left\langle f, \mathcal{W}_{p}\right\rangle\right|\left|\sum_{m=1}^{\infty} \lambda_{m n} \overline{\lambda_{m p}}\right| \\
& \leq\left(\sum_{n=1}^{\infty} \sum_{p \neq n}\left|\left\langle\mathcal{W}_{n}, f\right\rangle\right|^{2}\left|\sum_{m=1}^{\infty} \lambda_{m n} \overline{\lambda_{m p}}\right|\right)^{1 / 2} \cdot\left(\sum_{n=1}^{\infty} \sum_{p \neq n}\left|\left\langle f, \mathcal{W}_{p}\right\rangle\right|^{2}\left|\sum_{m=1}^{\infty} \lambda_{m n} \overline{\lambda_{m p}}\right|\right)^{1 / 2} .
\end{aligned}
$$

On solving the second term in the last product, we have

$$
\sum_{n=1}^{\infty} \sum_{p \neq n}\left|\left\langle f, \mathcal{W}_{p}\right\rangle\right|^{2}\left|\sum_{m=1}^{\infty} \lambda_{m n} \overline{\lambda_{m p}}\right|=\sum_{p=1 n \neq p}^{\infty} \sum\left|\left\langle f, \mathcal{W}_{p}\right\rangle\right|^{2}\left|\sum_{m=1}^{\infty} \lambda_{m n} \overline{\lambda_{m p}}\right|=\sum_{n=1}^{\infty} \sum_{p \neq n}\left|\left\langle\mathcal{W}_{n}, f\right\rangle\right|^{2}\left|\sum_{m=1}^{\infty} \lambda_{m n} \overline{\lambda_{m p}}\right|
$$

Thus,

$$
\left|R_{2}(f)\right| \leq \sum_{n=1}^{\infty} \sum_{p \neq n}\left|\left\langle\mathcal{W}_{n}, f\right\rangle\right|^{2}\left|\sum_{m=1}^{\infty} \lambda_{m n} \overline{\lambda_{m p}}\right|
$$

By (23), we have

$$
\begin{aligned}
\sum_{m=1}^{\infty}\left|\left\langle\gamma_{m}, f\right\rangle\right|^{2} & \geq \sum_{m=1}^{\infty} \sum_{n=1}^{\infty}\left|\lambda_{m n}\right|^{2}\left|\left\langle\mathcal{W}_{n}, f\right\rangle\right|^{2}-\sum_{n=1}^{\infty} \sum_{p \neq n}\left|\left\langle\mathcal{W}_{n}, f\right\rangle\right|^{2}\left|\sum_{m=1}^{\infty} \lambda_{m n} \overline{\lambda_{m p}}\right| \\
& =\sum_{n=1}^{\infty}\left|\left\langle\mathcal{W}_{n}, f\right\rangle\right|^{2}\left(\sum_{m=1}^{\infty}\left|\lambda_{m n}\right|^{2}-\sum_{p \neq n}\left|\sum_{m=1}^{\infty} \lambda_{m n} \overline{\lambda_{m p}}\right|\right) \\
& \geq k \sum_{n=1}^{\infty}\left|\left\langle\mathcal{W}_{n}, f\right\rangle\right|^{2} .
\end{aligned}
$$

Thus,

$$
k \mathcal{K}\|f\|^{2} \leq \sum_{m=1}^{\infty}\left|\left\langle\gamma_{m}, f\right\rangle\right|^{2} .
$$

Similarly, one can prove the upper frame condition.

\section{References}

[1] Coifman, R.R., Meyer, Y. and Wickerhauser, M.V. (1992) Wavelet Analysis and Signal Processing. In: Ruskai, M.B., et al., Eds., Wavelets and Their Applications, Jones and Barlett, Boston, 153-178.

[2] Coifman, R.R., Meyer, Y. and Wickerhauser, M.V. (1992) Size Properties of Wavelet Packets. In: Ruskai, M.B., et al., 
Eds., Wavelets and Their Applications, Jones and Barlett, Boston, 453-547.

[3] Shen, Z. (1995) Non-Tensor Product Wavelet Packets in $L^{2}\left(\mathbb{R}^{s}\right)$. SIAM Journal on Mathematical Analysis, 26, 10611074. http://dx.doi.org/10.1137/S0036141093243642

[4] Chui, C.K. and Li, C. (1993) Nonorthogonal Wavelet Packets. SIAM Journal on Mathematical Analysis, 24, $712-738$. http://dx.doi.org/10.1137/0524044

[5] Yang, S. and Cheng, Z. (2000) A-Scale Multiple Orthogonal Wavelet Packets. Mathematica Applicata, 13, 61-65. (In Chinese)

[6] Chen, Q. and Cheng, Z. (2007) A Study on Compactly Supported Orthogonal Vector Valued Wavelets and Wavelet Packets. Chaos, Solitons and Fractals, 31, 1024-1034. http://dx.doi.org/10.1016/j.chaos.2006.03.097

[7] Cohen, A. and Daubechies, I. (1993) On the Instability of Arbitrary Biorthogonal Wavelet Packets. SIAM Journal on Mathematical Analysis, 24, 1340-1354. http://dx.doi.org/10.1137/0524077

[8] Feng, Z. and Chen, G. (2001) Nonorthogonal Wavelet Packets with r-Scaling Functions. Journal of Computational Analysis and Applications, 3, 317-330. http://dx.doi.org/10.1023/A:1012046206504

[9] Hernandez, E. and Weiss, G. (1996) A First Course on Wavelets. CRC Press, Boca Raton. http://dx.doi.org/10.1201/9781420049985

[10] Daubechies, I. (1992) Ten Lectures on Wavelets. SIAM, Philadelphia. http://dx.doi.org/10.1137/1.9781611970104

[11] Chen, D. (2000) On the Splitting Trick and Wavelet Frame Packets. SIAM Journal on Mathematical Analysis, 31, 726-739. http://dx.doi.org/10.1137/S0036141097323333

[12] Long, R. and Chen, W. (1997) Wavelet Basis Packets and Wavelet Frame Packets. Journal of Fourier Analysis and Applications, 3, 239-256. http://dx.doi.org/10.1007/BF02649111

[13] Long, R. and Chen, W. (1995) Biorthogonal Wavelet Bases on $\mathbb{R}^{d}$. Applied Computational Harmonic Analysis, 2, 230-242. http://dx.doi.org/10.1006/acha.1995.1016

[14] Behera, B. (2001) Multiwavelet Packets and Frame Packets of $L^{2}\left(\mathbb{R}^{d}\right)$. Proceedings of the Indian Academy of Sciences (Mathematical Sciences), 111, 439-463. http://dx.doi.org/10.1007/BF02829617 
Scientific Research Publishing (SCIRP) is one of the largest Open Access journal publishers. It is currently publishing more than 200 open access, online, peer-reviewed journals covering a wide range of academic disciplines. SCIRP serves the worldwide academic communities and contributes to the progress and application of science with its publication.

Other selected journals from SCIRP are listed as below. Submit your manuscript to us via either submit@scirp.org or Online Submission Portal.
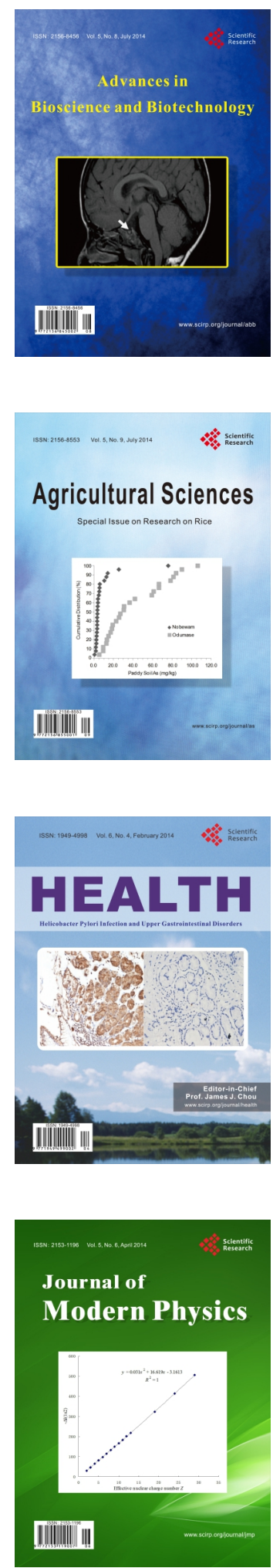
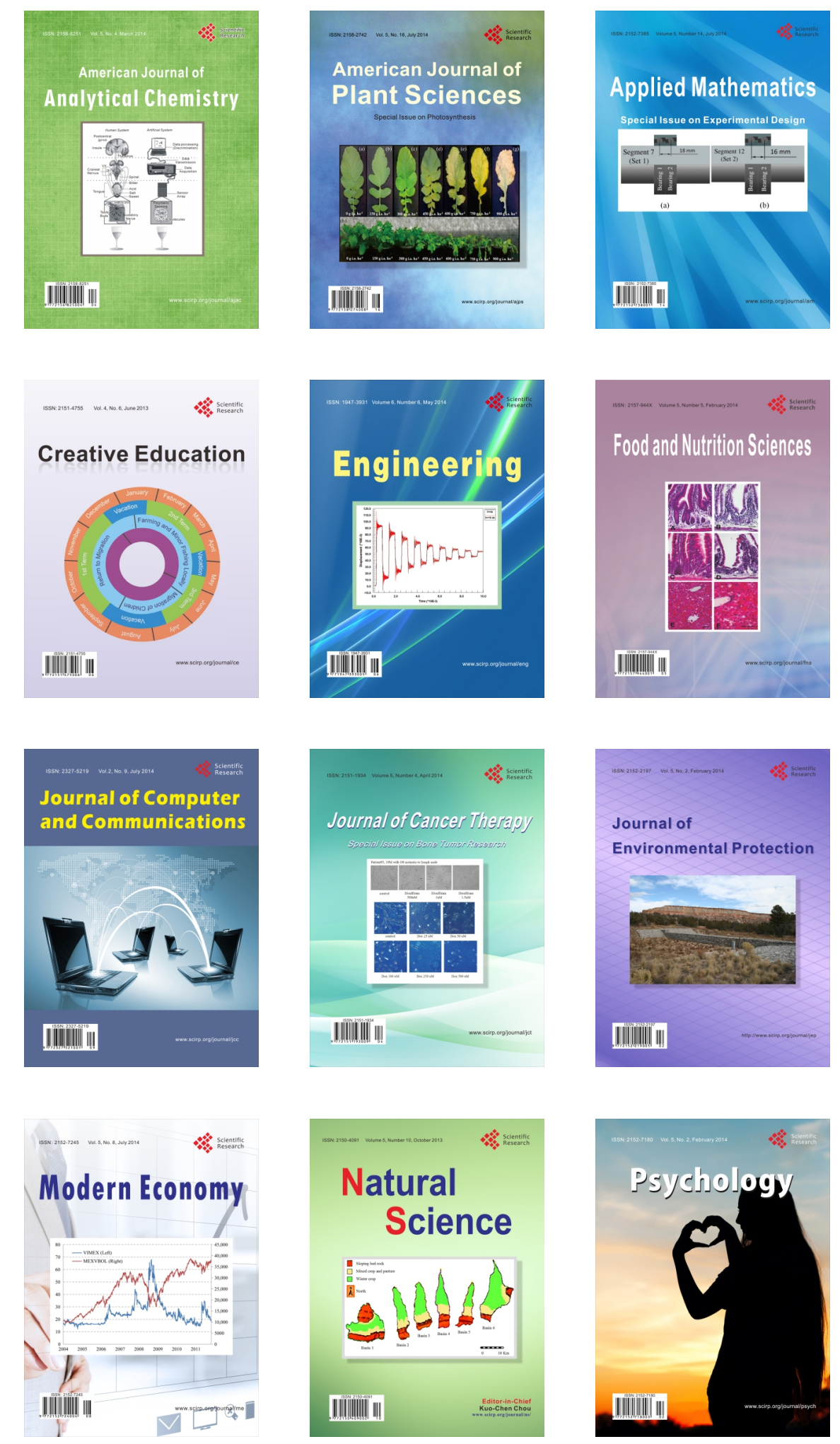